\title{
The tripartite motif-containing protein 3 on the proliferation and cytokine secretion of rheumatoid arthritis fibroblast-like synoviocytes
}

\author{
MINGJUN WANG ${ }^{*}$, JIAN WU* ${ }^{*}$ YUFAN GUO, XIN CHANG and TAO CHENG \\ Department of Rheumatology, The First Affiliated Hospital of Soochow University, Suzhou, Jiangsu 215000, P.R. China
}

Received November 28, 2016; Accepted January 18, 2017

DOI: $10.3892 / \mathrm{mmr} .2017 .6164$

\begin{abstract}
Recent studies have revealed fibroblast-like synoviocytes (FLS) as a pivotal effector cell in the inflamed joint of rheumatoid arthritis (RA) patients. FLS exhibit high proliferation rates and constitutive expression of cytokines, contributing to the pathogenesis of RA. In this study, we found that the expression of tripartite motif-containing protein 3 (TRIM3), a candidate tumor suppressor gene, was lower in synovial tissue samples of RA patients than in that of healthy controls. We then investigated the role of TRIM3 on the proliferation and cytokine secretion of primary cultured FLS from RA patients. Enforced expression of TRIM3 in RA FLS led to significantly decreased cell proliferation as indicated by Cell Counting Kit- 8 assay, reduced secretion of tumor necrosis factor- $\alpha$ (TNF)- $\alpha$, interleukin (IL)- $1 \beta$ and IL- 6 as indicated by enzyme-linked immunosorbent assays, and decreased p38 phosphorylation as assessed by western blot analysis. The proteins promoting cell cycles (cyclin D1 and PCNA) were downregulated and the protein negatively regulating cell cycle progression (p53 and p21) was upregulated after TRIM3 overexpression. Importantly, TRIM3 knockdown had reverse effects on cell proliferation, which was suppressed by the $\mathrm{p} 38$-specific inhibitor SB203580. In conclusion, the current results demonstrated the downregulation of TRIM3 expression in RA synovial tissues. Importantly, TRIM3 exerted an anti-proliferation role in RA FLS via p38 signaling pathway.
\end{abstract}

\section{Introduction}

Rheumatoid arthritis (RA) is a chronic autoimmune disease, which primarily affects small joints of the hands and feet. The

Correspondence to: Dr Tao Cheng, Department of Rheumatology, The First Affiliated Hospital of Soochow University, 188 Shixi Street, Suzhou, Jiangsu 215000, P.R. China

E-mail: hp2131@163.com

*Contributed equally

Key words: rheumatoid arthritis, tripartite motif-containing protein 3, proliferation, p38, cytokine pathological characteristic of RA includes synovial hyperplasia and inflammation, pannus formation, cartilage loss and joint destruction (1). Fibroblast-like synoviocytes (FLS), a type of synovial lining cell, is considered as a pivotal effector cell in the inflamed joint. FLS exhibit high proliferation rates, constitutive expression of cytokines, and anchorage-independent cell growth (2-5). The hyperplasia of FLS leads to pannus formation and joint destruction (6). The production of cytokines, such as tumor necrosis factor (TNF)- $\alpha$, interleukin (IL)-1 $\beta$ and IL-6, causes the erosion of cartilage and contributes to pathogenesis of RA (7).

Tripartite motif-containing protein 3 (TRIM3) belongs to TRIM proteins family, which regulates diverse biological functions including innate immune response, development and carcinogenesis $(8,9)$. Emerging evidence has suggested that TRIM3 is a candidate tumor suppressor gene for glioblastomas (10-12) and colorectal cancer (13). Overexpression of TRIM3 inhibited cell proliferation of cancer cells $(13,14)$. However, little is known about the expression and function of TRIM proteins, including TRIM3 in RA progression.

In the present study, we found that the mRNA and protein levels of TRIM3 were significantly decreased in RA synovial tissues. TRIM3 exerted an anti-proliferation role in primary cultured FLS from RA patients via p38 signaling pathway. Taken together, results of the current study suggest TRIM3 may be a candidate therapeutic target for RA.

\section{Materials and methods}

Tissue specimens. A total of $30 \mathrm{RA}$ patients and 12 joint trauma patients (healthy control) were enrolled in this study. RA patients were diagnosed according to the American College of Rheumatology Criteria for Classification of RA (15). Written informed consent was obtained from all patients, and the study was approved by the Ethics Committee of the First Affiliated Hospital of Soochow University. Synovial tissue samples were obtained during joint replacement surgery, snap-frozen and stored at $-80^{\circ} \mathrm{C}$.

Real-time polymerase chain reaction (PCR). Total RNA was isolated from collected synovial tissue samples using TRIzol (Invitrogen, Carlsbad, CA, USA) according to the manufacturer's protocol. After treating with RNase-free DNase (Roche Diagnostics, Indianapolis, IN, USA) to remove any residual 
genomic DNA, total RNA ( $2 \mu \mathrm{g})$ was reverse-transcribed using cDNA synthesis kit (Thermo Fisher Scientific, Inc., Rockford, IL, USA). TRIM3 mRNA levels were examined by real-time PCR with SYBR-Green qPCR Master Mixes (Thermo Fisher Scientific Inc.) on an ABI 7300 cycler (Applied Biosystems,FosterCity,CA,USA).Glyceraldehyde-3-phosphate dehydrogenase (GAPDH) mRNA levels were used for normalization. The primers were as follows: TRIM3, 5'-GAGGGCAA GTTCAAGACCAAG-3' and 5'-GGAAGGTAAAGACGC AGCAAG-3'; GAPDH, 5'-CACCCACTCCTCCACCTTTG-3' and 5'-CCACCACCCTGTTGCTGTAG-3'. TRIM3 mRNA expression was calculated using the $\mathrm{CT}$ method as previously described (16).

Western blot analysis. Protein were extracted from frozen tissue samples and cells by using RIPA buffer in the presence of proteinase inhibitor cocktail (Sigma, St. Louis, MO, USA). Protein concentrations were determined using the BCA kit (Thermo Fisher Scientific, Inc.). Equal amounts of protein from samples were separated on $10 \%$ SDS-PAGE and then transferred to a nitrocellulose membrane (Millipore Corp., Bedford, MA, USA). For western blot analysis, the membrane was pre-incubated with $5 \%$ skimmed milk in TBST (Tris-buffered saline containing $0.1 \%$ Tween-20) at $25^{\circ} \mathrm{C}$ for $1 \mathrm{~h}$, and then incubated with primary antibodies overnight at $4^{\circ} \mathrm{C}$. After being washed three times in TBST, horseradish peroxidase-conjugated secondary antibodies (Beyotime Institute of Biotechnology, Shanghai, China) were added and incubated for $1 \mathrm{~h}$ at $25^{\circ} \mathrm{C}$. After three washes in TBST, hybridized bands were detected using the ECL detection kit (Millipore Corp.). The sources of primary antibodies were as follows: antibodies against TRIM3, cyclin D1, and p21 were from Abcam (Cambridge, MA, USA); antibodies to PCNA, p53, p-p38, p38 and GAPDH were from Cell Signaling Technology, Inc. (Danvers, MA, USA).

Isolation of FLS cell lines from RA patients. FLS cell lines were prepared from the synovium of RA patients as previously described (17). In brief, synovial tissues were minced into $1-\mathrm{mm}$ pieces and treated with $0.4 \%$ type 1 collagenase (Sigma) and DNase in Dulbecco's modified Eagle's medium (DMEM; Hyclone, Logan, UT, USA) for $2 \mathrm{~h}$ at $37^{\circ} \mathrm{C}$ in a $5 \% \mathrm{CO}_{2}$-humidified incubator. Undigested tissues were removed with a cell strainer.

The dissociated cells were centrifuged at 1,000 rpm for 10 min, resuspended in DMEM supplemented with $10 \%$ fetal bovine serum (FBS; Gibco, Carlsbad, CA, USA), $100 \mathrm{U} / \mathrm{ml}$ penicillin, and $100 \mu \mathrm{g} / \mathrm{ml}$ streptomycin and seeded in $10-\mathrm{cm}$ culture plates. Medium was replaced every 2-3 days to remove suspended cells, and cells were sub-cultured when the primary culture reached confluence. After the third passage, the cells exhibited characteristic morphology of fibroblasts and were verified by immunocytochemistry as a homogeneous population ( $>95 \%$ vimentin-positive and CD68-negative (5); Fig. 1). FLS at passages 4-8 were used for the experiments.

Lentivirus production and RNA interference. To create TRIM3 lentiviral expression construct, the full length human
TRIM3 cDNA was inserted into pLVX-puro (Clontech Laboratories, Inc., Palo Alto, CA, USA) and verified by sequencing. pLVX-puro or pLVX-puro-TRIM3 together with viral packaging plasmids (psPAX2 and pMD2G) was transfected into HEK293T cells using Lipofectamine 2000 (Invitrogen) according to the manufacturer's protocol. Vector or TRIM3 expression viral supernatant was harvested at $48 \mathrm{~h}$ after transfection and used to transduce FLS cell lines.

siRNA targeting human TRIM3 mRNA (5'-GCUCACUG UCACUACCAAA-3') and a non-specific scramble siRNA sequence (siNC) were synthesized by GenePharma (Shanghai, China) and transfected into FLS cells using Lipofectamine 2000 (Invitrogen) according to the manufacturer's instructions.

Experimental grouping. To investigate the function of TRIM3 in RA, the FLS cells were divided into three groups: group 1 (Mock), cells were cultured without any treatment; group 2, cells were infected with pLVX-puro vector virus; group 3, cells were infected with TRIM3-expressing virus. Cell Counting Kit-8 (CCK-8) assays were carried out at 0,1 , 2 and 3 days after viral infection. Western blot analysis and enzyme-linked immunosorbent assays were performed at $48 \mathrm{~h}$ after viral infection.

To further explore the involvement of p38 signaling, the FLS cells were divided into five groups: group 1 (Mock), cells were cultured without any treatment; group 2, cells were pretreated with DMSO for $1 \mathrm{~h}$ and then transfected with control siRNA (siNC); group 3, cells were pretreated with $10 \mu \mathrm{M}$ p38 inhibitor (SB203580; Selleck Chemicals, Houston, TX, USA) for $1 \mathrm{~h}$ and then transfected with siNC; group 4, cells were pretreated with DMSO for $1 \mathrm{~h}$ and then transfected with TRIM3 siRNA (siTRIM3); group 5, cells were pre-treated with $10 \mu \mathrm{M} \mathrm{SB} 203580$ for $1 \mathrm{~h}$ and then transfected with siTRIM3. CCK-8 assays were carried out at $0,1,2$ and 3 days after siRNA transfection. Western blot analysis was performed at $48 \mathrm{~h}$ after siRNA transfection.

Cell proliferation assay. FLS proliferation was determined with the CCK-8 kit (Signalway Antibody LLC (SAB), College Park, MD, USA). FLS cells $\left(3 \times 10^{3} /\right.$ well) were plated into 96-well plates. After cultured overnight, the cells were treated as indicated and cultured at $37^{\circ} \mathrm{C}$ for $0,1,2$ and 3 days. After the incubation period, the cultured media were replaced with $10 \%$ CCK-8 reagent (in DMEM), and the cells were incubated at $37^{\circ} \mathrm{C}$ for another $1 \mathrm{~h}$. The absorbance at $450 \mathrm{~nm}$ was read with a microplate reader (Bio-Rad Laboratories, Inc., Hercules, CA, USA).

Immunoassays of $I L-1 \beta, I L-6$ and TNF- $\alpha$. The amounts of secreted cytokines in culture media were measured by ELISA. Assays were performed following the manufacturer's instructions (Bio-Swamp Life Science, Shanghai, China). The absorbance was read at $450 \mathrm{~nm}$ and recorded on a microplate reader (Bio-Rad Laboratories, Inc.).

Statistical analysis. The results are presented as the mean \pm SD. One-way analysis of variance followed by Dunnett's multiple comparisons was used for statistical analysis by GraphPad Prism 6.0 software (GraphPad, San Diego, CA, USA). $P<0.05$ was considered significant. 
CD68

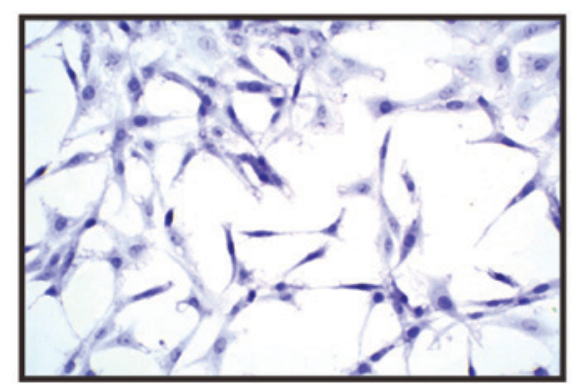

Vimentin

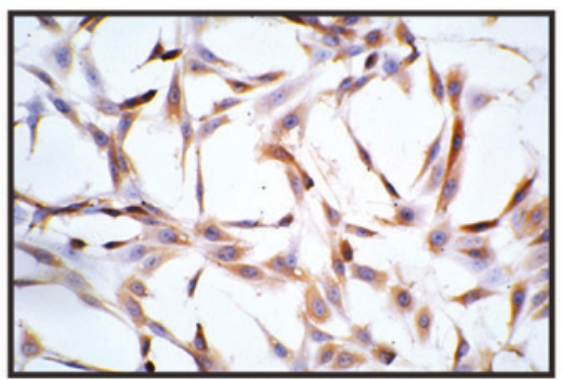

Figure 1. Identification of primary cultured FLS. FLS cultured in the coverslips were immunocytochemically stained with antibodies against CD68 or vimentin, and counter-stained with hematoxylin (DAB, brown; hematoxylin, blue). More than 95\% of the cells were vimentin-positive and CD68-negative. FLS, fibroblast-like synoviocytes.

A

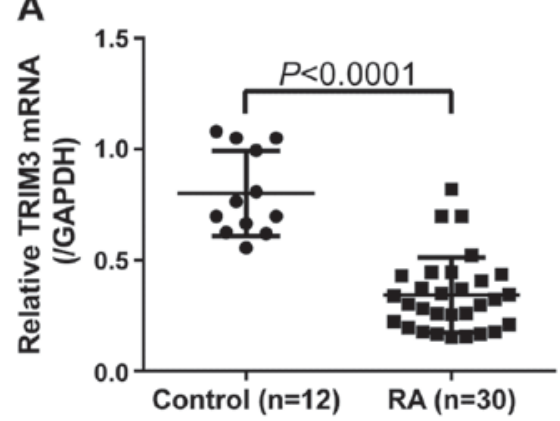

B

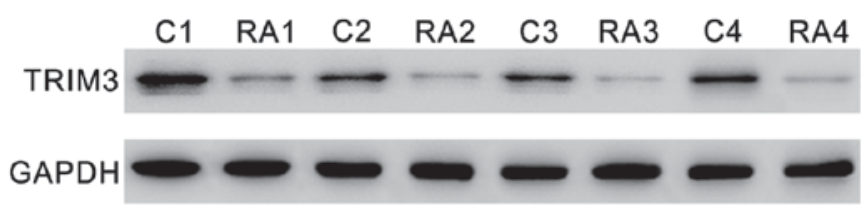

Figure 2. TRIM3 expression in synovial tissues. (A) mRNA levels of TRIM3 in synovial tissue samples from RA patients (n=30) and healthy control (n=12) were detected by real-time PCR. (B) Protein levels of TRIM3 in RA synovial tissue samples (RA1-RA4) and healthy control (C1-C4) were determined by western blot analysis. TRIM3, tripartite motif-containing protein 3; RA, rheumatoid arthritis.

\section{Results}

TRIM3 is downregulated in synovial tissue samples of $R A$ patients. To determine TRIM3 expression in synovial tissues, synovial tissue specimens were collected from 30 RA patients and 12 joint trauma patients (healthy control). As indicated by real-time PCR analysis, compared with healthy control, the TRIM3 mRNA in synovial tissues of RA patients decreased by $57.2 \%$ (Fig. $2 \mathrm{~A}, \mathrm{P}<0.0001$ ). Western blot analysis also showed the downregulation of TRIM3 in RA tissues at translational level (Fig. 2B).

Inhibition of RA FLS cell proliferation by TRIM3 overexpression. Synoviocyte hyperplasia is the most prominent characteristic of RA (6). To investigate the function of TRIM3 in RA, FLS cells were isolated from the synovium of RA patients and TRIM3 was overexpressed in RA FLS cells by lentiviral transduction. CCK- 8 assays showed that TRIM3 overexpressing virus (pLVX-puro-TRIM3) significantly inhibited the proliferation of RA FLS cells at 1, 2 and 3 days as compared to untreated cells (Mock) and cells transduced with vector virus (pLVX-puro) (Fig. 3A). The overexpression of TRIM3 in virally transduced cells was confirmed by western blot analysis at $48 \mathrm{~h}$ after lentiviral transduction (Fig. 3B).

The expression levels of cell proliferation regulators, cyclin D1, PCNA, p53 and p21, were also evaluated by western blot analysis. The levels of PCNA (a marker of cell proliferation) (18) and cyclin D1 (a cell cycle-promoting protein) (19) were downregulated, while the levels of p53 (a growth suppressor protein) (20) and its downstream target gene, p21, was upregulated by TRIM3 overexpression (Fig. 3B). These results suggested that TRIM3 inhibited the proliferation of RA FLS cells.

Suppression effect of TRIM3 on the secretion of cytokines from RA FLS. Cytokines, such as TNF- $\alpha$, IL-1 $\beta$ and IL-6, contribute to the pathogenesis of RA (7). We next measured the changes of three cytokines in culture medium conditioned with RA FLS after overexpression of TRIM3 (Fig. 4). All cytokine levels were significantly decreased in the presence of TRIM3 overexpression.

TRIM3 inhibits the proliferation of RA FLS via p38 signaling. p38 is a key signaling component of inflammatory disease including RA (21). To further investigate the molecular mechanism through which TRIM3 inhibited the growth of RA FLS, western blot analyses were performed to study changes in p38 signaling. As shown in Fig. 5, p-p38 levels were significantly decreased at $48 \mathrm{~h}$ after TRIM3 expressing viral infection, while total p38 levels were unchanged.

To further explore the involvement of p38 signaling in suppression effect of TRIM3 on the proliferation of RA FLS, the p38-specific inhibitor SB203580 was added before TRIM3 siRNA transfection. Cell proliferation was repressed by SB203580 treatment, but promoted by TRIM3 knockdown. Additionally, SB203580 treatment reduced the promotion effects of TRIM3 on FLS proliferation (Fig. 6A). 


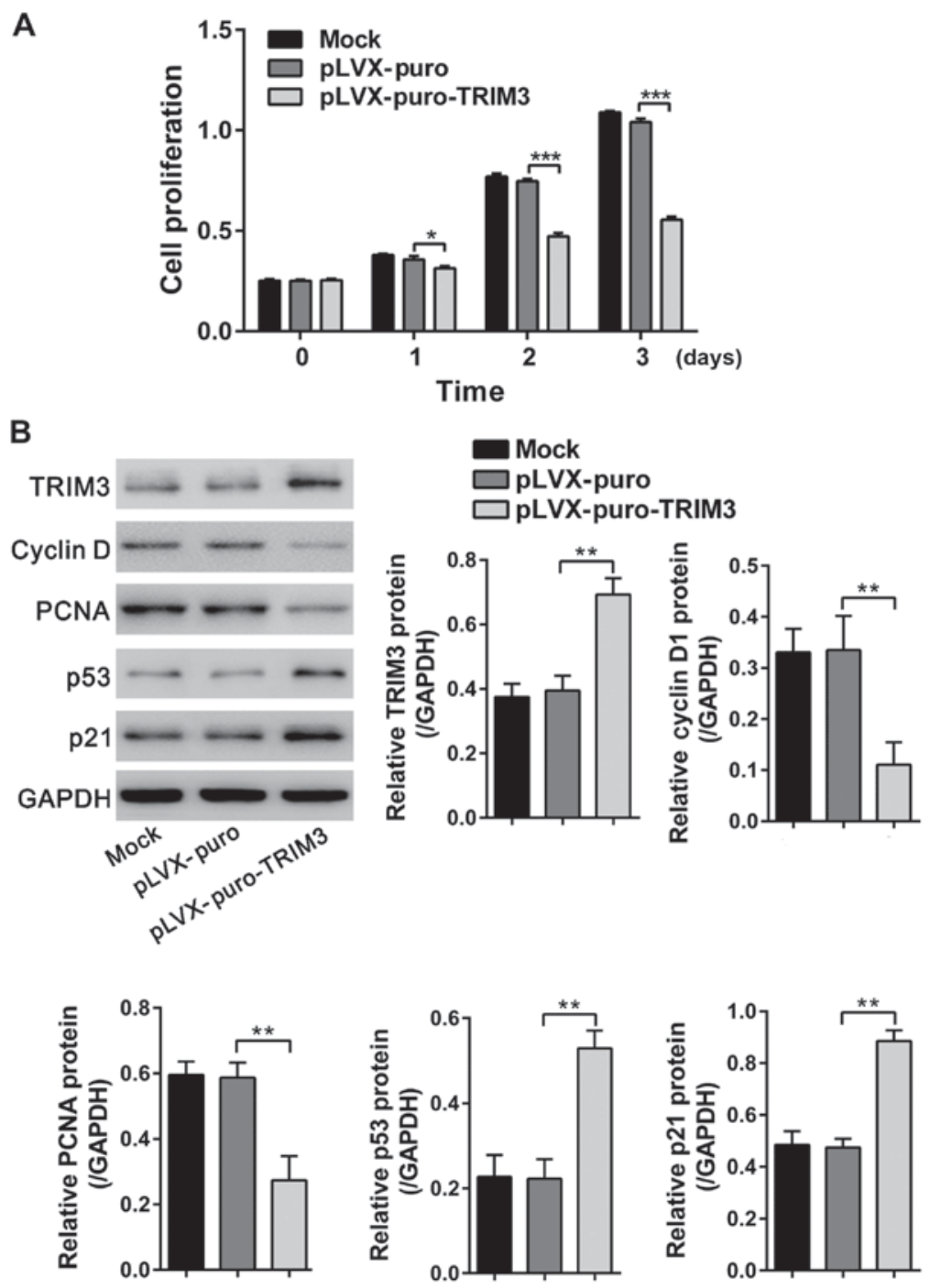

Figure 3. Inhibition of RA FLS cell proliferation by TRIM3 overexpression. (A) Results of CCK-8 assays following infection of pLVX-puro or pLVX-puro-TRIM3 lentivirus into RA FLS cells. Proliferation was significantly suppressed by pLVX-puro-TRIM3 viral transduction. (B) Expression of TRIM3, cyclin D1, PCNA, p53 and p21 was evaluated by western blot analysis at $48 \mathrm{~h}$ after viral transduction. Data are based on three independent experiments, and shown as mean \pm SD. ${ }^{*} \mathrm{P}<0.05,{ }^{* *} \mathrm{P}<0.01,{ }^{* * * *} \mathrm{P}<0.001$. RA, rheumatoid arthritis; FLS, fibroblast-like synoviocytes; TRIM3, tripartite motif-containing protein 3; CCK-8, Cell Counting Kit-8.
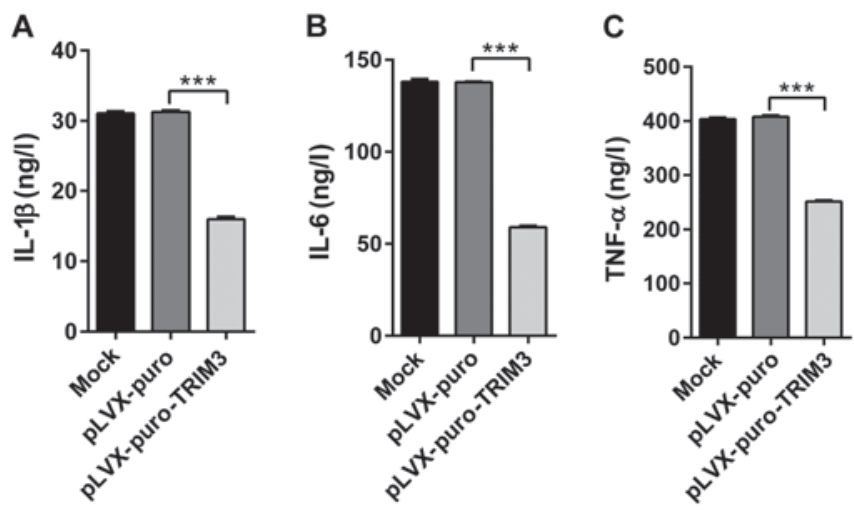

Figure 4. Suppression effect of TRIM3 on the secretion of cytokines from RA FLS. Results of ELISA assays following infection of pLVX-puro or pLVX-puro-TRIM3 lentivirus into RA FLS cells. Levels of (A) IL-1 $\beta$, (B) IL-6 and (C) TNF- $\alpha$ were significantly suppressed at 48 h after pLVX-puro-TRIM3 viral transduction. ${ }^{* * *} \mathrm{P}<0.001$. TRIM3, tripartite motif-containing protein 3; RA, rheumatoid arthritis; FLS, fibroblast-like synoviocytes; IL, interleukin; TNF- $\alpha$, tumor necrosis factor- $\alpha$.

The protein levels of TRIM3, p-p38, p38, cyclin D1, PCNA, p53 and 21 were then assessed by western blot analysis. As shown in Fig. 6B, SB203580 blocked the TRIM3 knockdown mediated increase of p-p38, cyclin D1 and PCNA, and rescued 


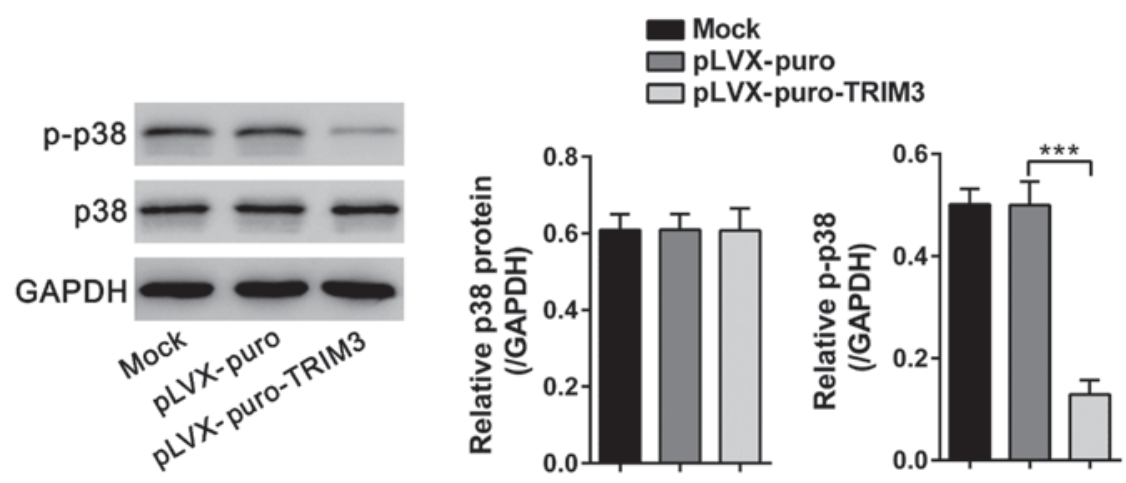

Figure 5. TRIM3 inactivates p38 signaling. Expression of p-p38 and p38 was evaluated by western blot analysis at $48 \mathrm{~h}$ after viral transduction. Data are based on three independent experiments, and shown as mean $\pm \mathrm{SD}$. ${ }^{* * *} \mathrm{P}<0.001$. TRIM3, tripartite motif-containing protein 3 .

A

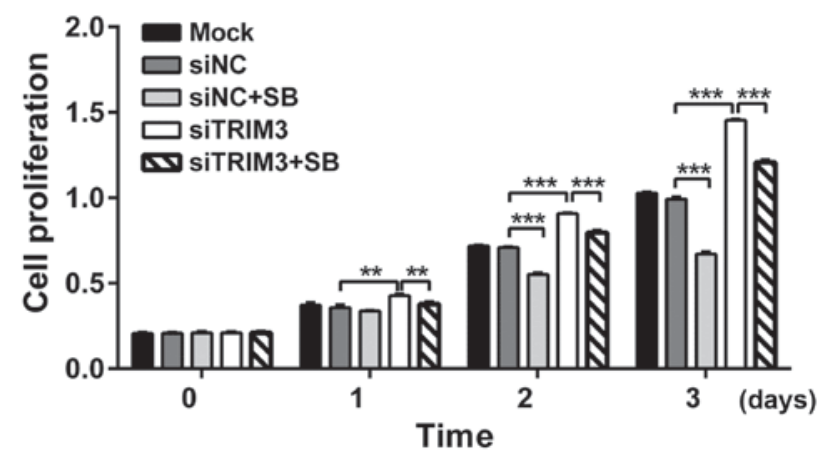

B
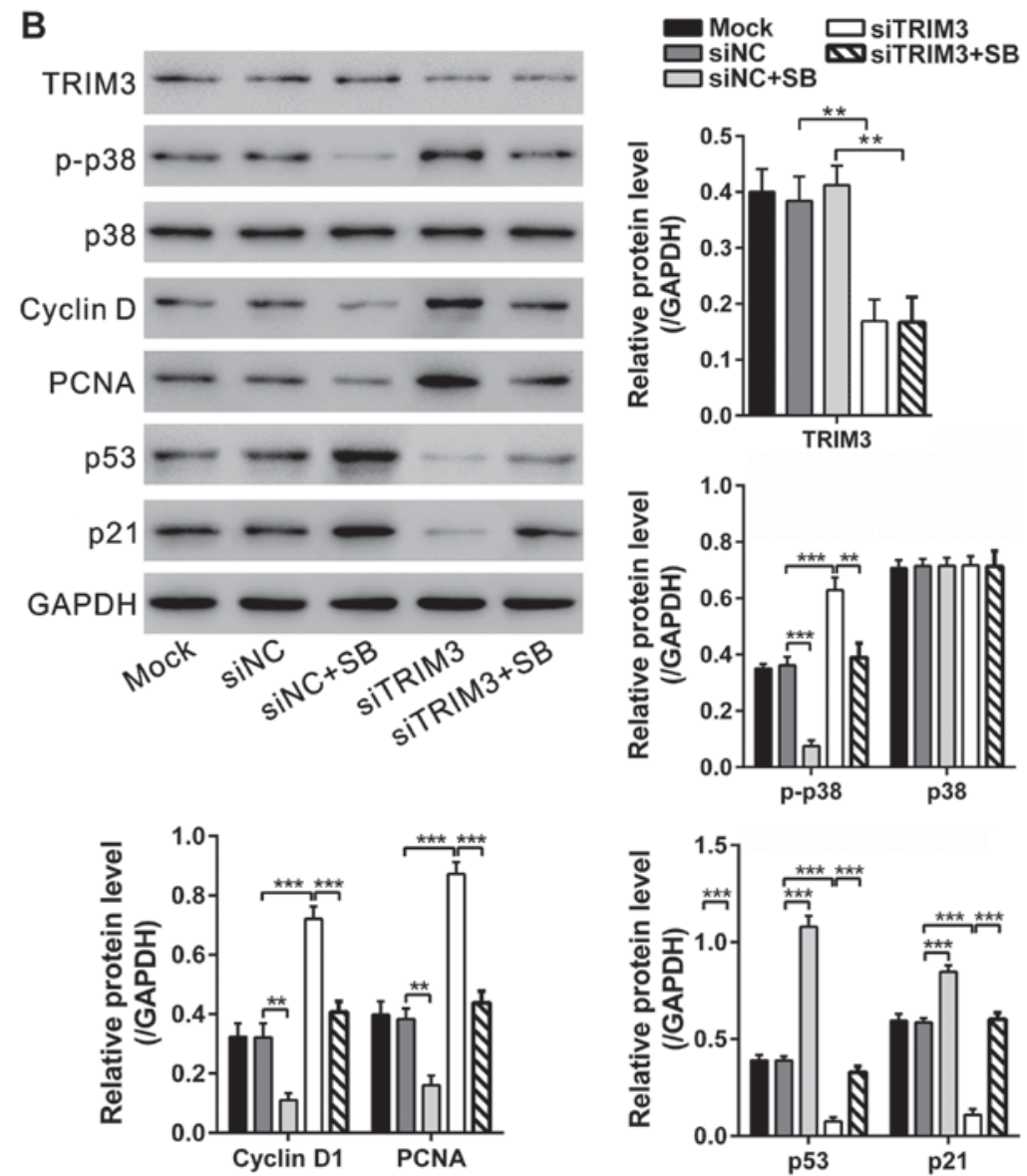

Figure 6. TRIM3 inhibits the proliferation of RA FLS via p38 signaling. The FLS cells were pre-treated with DMSO or $10 \mu \mathrm{M} \mathrm{SB} 203580$ for $1 \mathrm{~h}$ and then transfected with control siRNA (siNC) or TRIM3 siRNA (siTRIM3). Cells cultured without any treatment were set as control (Mock). (A) CCK-8 assays were performed at 0, 1, 2 and 3 days after siRNA transfection. (B) Expression of TRIM3, p-p38, p38, cyclin D1, PCNA, p53 and p21 was evaluated by western blot analysis at $48 \mathrm{~h}$ after treatment. Data are based on three independent experiments, and shown as mean $\pm \mathrm{SD}$. ${ }^{* *} \mathrm{P}<0.01$; ${ }^{* * * *} \mathrm{P}<0.001$. TRIM3, tripartite motif-containing protein 3; RA, rheumatoid arthritis; FLS, fibroblast-like synoviocytes; siTRIM3, TRIM3 siRNA; CCK-8, Cell Counting Kit-8. 
the TRIM3 knockdown mediated decrease of p53 and p21. Collectively, these results support the hypothesis that the suppression effects of TRIM3 on RA FLS proliferation may be achieved via inactivating p38 signaling.

\section{Discussion}

In the present study, we show that the expression of TRIM3 was significantly lower in RA synovial tissues than in control tissues. Overexpression of TRIM3 in RA FLS led to decreased cell proliferation and reduced production of TNF- $\alpha, \mathrm{IL}-1 \beta$ and IL-6. The suppression effects of cell proliferation by TRIM3 were partially mediated via impaired $\mathrm{p} 38$ signaling.

Pathologic expression of TRIM3 has been identified in glioblastomas (10-12), colorectal cancer (13) and hepatocellular carcinoma, where its expression levels are lower than in normal tissues. TRIM3 functions as a tumor suppressor (10-13). Studies of cancer cells have also shown that TRIM3 overexpression can inhibit cell proliferation $(13,14)$. The present results of CCK-8 and western blot (PCNA and cyclin D1) analyses in RA FLS were in accordance with previous findings.

It has been reported that RA synovium expressed many cytokines (22). Among these cytokines, IL-1 $\beta$, IL- 6 and TNF- $\alpha$ are critical in the evolution of RA, partly because anti-cytokine strategies have been successfully applied for patients $(23,24)$. Several members of TRIMs, such as TRIM30 $\alpha$, TRIM8 and TRIM21, are involved in the innate immunity response (8). The current study revealed that TRIM3 overexpression can inhibit the secretion of IL-1 $\beta$, IL- 6 and TNF- $\alpha$, indicating the role of TRIM3 in inflammatory response and the potential clinical application of TRIM3 in RA.

Overexpression of TRIM3 in colorectal cancer cells is able to upregulate p53 with concomitant induction of downstream target genes, p21 and GADD45 (13). p38, a cell proliferation regulator, is a key signaling component of inflammatory disease including RA (21). Chemotherapeutic agents can activate 553 via $\mathrm{p} 38$ signaling (25). Here, TRIM3 overexpression decreased the expression of p53, p21 and p-p38 in RA FLS. By using the p38-specific inhibitor SB203580, we found that the effects of TRIM3 on p53 was dependent on p38 activity. SB203580 blocked the effects of TRIM3 knockdown that increased cell proliferation, and enhanced the expression of cyclin D1 and PCNA. Therefore, the collective evidence indicated that TRIM3 may inactivate p38/p53 signaling pathway and could serve as a potential therapeutic target.

In conclusion, our findings demonstrated that TRIM3 expression was downregulated in RA synovium. It inhibited RA FLS proliferation via p38 signaling pathway, and suppressed cytokine secretion. Thus, TRIM3 plays a potentially protective role for FLS hyperplasia and inflammation.

\section{References}

1. Majithia V and Geraci SA: Rheumatoid arthritis: Diagnosis and management. Am J Med 120: 936-939, 2007.

2. Ritchlin C, Dwyer E, Bucala R and Winchester R: Sustained and distinctive patterns of gene activation in synovial fibroblasts and whole synovial tissue obtained from inflammatory synovitis. Scand J Immunol 40: 292-298, 1994.

3. Bucala R, Ritchlin C, Winchester R and Cerami A: Constitutive production of inflammatory and mitogenic cytokines by rheumatoid synovial fibroblasts. J Exp Med 173: 569-574, 1991.
4. Lafyatis R, Remmers EF, Roberts AB, Yocum DE, Sporn MB and Wilder RL: Anchorage-independent growth of synoviocytes from arthritic and normal joints. Stimulation by exogenous platelet-derived growth factor and inhibition by transforming growth factor-beta and retinoids. J Clin Invest 83: 1267-1276, 1989.

5. Bartok B and Firestein GS: Fibroblast-like synoviocytes: Key effector cells in rheumatoid arthritis. Immunol Rev 233: 233-255, 2010.

6. Wicks I, Cooley H and Szer J: Autologous hemopoietic stem cell transplantation: A possible cure for rheumatoid arthritis? Arthritis Rheum 40: 1005-1011, 1997.

7. Firestein GS: Invasive fibroblast-like synoviocytes in rheumatoid arthritis. Passive responders or transformed aggressors? Arthritis Rheum 39: 1781-1790, 1996.

8. Ozato K, Shin DM, Chang TH and Morse HC 3rd: TRIM family proteins and their emerging roles in innate immunity. Nat Rev Immunol 8: 849-860, 2008.

9. Hatakeyama S: TRIM proteins and cancer. Nat Rev Cancer 11: 792-804, 2011.

10. Liu Y, Raheja R, Yeh N, Ciznadija D, Pedraza AM, Ozawa T, Hukkelhoven E, Erdjument-Bromage H, Tempst P, Gauthier NP, et al: TRIM3, a tumor suppressor linked to regulation of p21 Waf1/Cipl. Oncogene 33: 308-315, 2014.

11. Chen G, Kong J, Tucker-Burden C, Anand M, Rong Y, Rahman F, Moreno CS, Van Meir EG, Hadjipanayis CG and Brat DJ: Human Brat ortholog TRIM3 is a tumor suppressor that regulates asymmetric cell division in glioblastoma. Cancer Res 74: 4536-4548, 2014.

12. Boulay JL, Stiefel U, Taylor E, Dolder B, Merlo A and Hirth F: Loss of heterozygosity of TRIM3 in malignant gliomas. BMC Cancer 9: 71, 2009.

13. Piao MY, Cao HL, He NN, Xu MQ, Dong WX, Wang WQ, Wang BM and Zhou B: Potential role of TRIM3 as a novel tumour suppressor in colorectal cancer (CRC) development. Scand J Gastroenterol 51: 572-582, 2016.

14. Raheja R, Liu Y, Hukkelhoven E, Yeh N and Koff A: The ability of TRIM3 to induce growth arrest depends on RING-dependent E3 ligase activity. Biochem J 458: 537-545, 2014.

15. Cohen S and Emery P: The American College of Rheumatology/European League Against Rheumatism criteria for the classification of rheumatoid arthritis: A game changer. Arthritis Rheum 62: 2592-2594, 2010.

16. Saha SK, Roy S and Khuda-Bukhsh AR: Ultra-highly diluted plant extracts of Hydrastis canadensis and Marsdenia condurango induce epigenetic modifications and alter gene expression profiles in HeLa cells in vitro. J Integr Med 13: 400-411, 2015.

17. Nishida K, Komiyama T, Miyazawa S, Shen ZN, Furumatsu T, Doi H, Yoshida A, Yamana J, Yamamura M, Ninomiya Y, et al: Histone deacetylase inhibitor suppression of autoantibody-mediated arthritis in mice via regulation of $\mathrm{p} 16^{\mathrm{INK} 4 \mathrm{a}}$ and p21 $1^{\text {WAF1/Cipl }}$ expression. Arthritis Rheum 50: 3365-3376, 2004.

18. Robbins BA, de la Vega D, Ogata K, Tan EM and Nakamura RM: Immunohistochemical detection of proliferating cell nuclear antigen in solid human malignancies. Arch Pathol Lab Med 111: 841-845, 1987.

19. Fu M, Wang C, Li Z, Sakamaki T and Pestell RG: Minireview: Cyclin D1: Normal and abnormal functions. Endocrinology 145: 5439-5447, 2004.

20. Montenarh M: Functional implications of the growth-suppressor oncoprotein p53 (Review). Int J Oncol 1: 37-45, 1992.

21. Pargellis C and Regan J: Inhibitors of p38 mitogen-activated protein kinase for the treatment of rheumatoid arthritis (Review). Curr Opin Investig Drugs 4: 566-571, 2003.

22. McInnes IB and Schett G: Cytokines in the pathogenesis of rheumatoid arthritis. Nat Rev Immunol 7: 429-442, 2007.

23. Nishimoto N, Hashimoto J, Miyasaka N, Yamamoto K, Kawai S, Takeuchi T, Murata N, van der Heijde D and Kishimoto T: Study of active controlled monotherapy used for rheumatoid arthritis, an IL-6 inhibitor (SAMURAI): Evidence of clinical and radiographic benefit from an $\mathrm{x}$ ray reader-blinded randomised controlled trial of tocilizumab. Ann Rheum Dis 66: 1162-1167, 2007.

24. Maini RN and Taylor PC: Anti-cytokine therapy for rheumatoid arthritis. Annu Rev Med 51: 207-229, 2000.

25. Sanchez-Prieto R, Rojas JM, Taya Y and Gutkind JS: A role for the p38 mitogen-acitvated protein kinase pathway in the transcriptional activation of 553 on genotoxic stress by chemotherapeutic agents. Cancer Res 60: 2464-2472, 2000. 